\title{
A MULTIFRACTAL ANALYSIS OF LIDAR MEASURED WATER VAPOUR
}

\author{
J. E. PINZÓN* , C. E. PUENTE, M. B. PARLANGE ${ }^{* *}$ and W. EICHINGER ${ }^{* * *}$ \\ Hydrologic Science, University of California, Davis, CA 95616, USA
}

(Received in final form 1 August, 1995)

\begin{abstract}
Accurate and fast-response measurements of space-time observations of specific humidity were made above a drying land surface at the University of California at Davis, using the Los Alamos water Raman-lidar. In an attempt to quantify the space-time intermittency features of turbulent flows in the lower atmosphere, a multifractal analysis of these water vapour measurements was performed. The structure of the specific humidity, $\theta(x, t)$, was analyzed quantifying a scalar gradient measure ( $\sim$ grad $\left.\theta]^{2}\right)$ both in time and space, for all possible one-dimensional cuts, i.e. $\chi_{t}(x, t)=[\partial \theta(x, t) / \partial t]^{2}$ and $\chi_{x}(x, t)=[\partial \theta(x, t) / \partial x]^{2}$. The results confirm the multifractal nature of this scalar gradient measure (a type of scalar dissipation rate) and show that humidity measurements at fixed times $\left(\chi_{x}\right)$ are more intermittent (e.g. have less entropy dimension) than those at fixed locations in space $\left(\chi_{t}\right)$. Similar multifractal behaviour of the spatial data, with and without a transformation from the observed wind velocities, supports the validity of Taylor's hypothesis for the studied fields.
\end{abstract}

\section{Introduction}

Atmospheric turbulence phenomena exhibit rich behaviour with highly spiked (singular) distributions in both time and space. The core of the turbulence problem is how to predict this complex behaviour. Unfortunately, it has been impossible to describe the details of the phenomena deterministically, and general stochastic theories do not exist for other than simple and oftentimes unrealistic conditions, e.g., ergodic, isotropic, homogeneous and stationary turbulence. Although several attempts have been made to extend the theories to the most general situations, no solution appears to be in sight, see e.g. Hunt et al. (1991), Sirovich (1991).

The singularity of the observed data is the main limitation encountered. This prevents a complete general (deterministic) characterization, and results in stochastic models that, even though sophisticated, have at least two limitations: (a) they approximate the observed singular patterns and consequently can track only some statistics of the processes, e.g. mean, variance, spatial correlations, power spectrum, multifractal spectrum, codimension function, etc., and (b) they are typically limited by their analytical tractability, e.g. the closure problem, etc. This work is concerned with the description of the intermittency seen in turbulence, as lately characterized in terms of multifractal properties, e.g. Meneveau and Sreenivasan (1987), Schertzer and Lovejoy (1989).

* Also at Institute of Theoretical Dynamics, University of California, Davis, CA 95616, USA.

** Also at Department of Biological and Agricultural Engineering, University of California, Davis, CA 95616, USA.

*** Also at Los Alamos National Laboratory, Los Alamos, NM 87545, USA. 
After Mandelbrot's $(1974,1983)$ pioneering work in fractal geometry, it has been recognized that the interwoven events that exist across different spatial scales may provide new insights into the structure of turbulence. For this purpose, phenomenological self-similar models have been developed which condense infinite degrees of freedom into a few relevant parameters. Sreenivasan et al. (1989) observed power laws in the dissipation rate of passive scalars $\left(\sim[\operatorname{grad} \theta]^{2}\right)$ in turbulent shears flows and they used their results to describe the intermittent fluctuations measured. They noted that the scalar dissipation rate obeys multifractal properties. Meneveau and Sreenivasan (1987) proposed for the energy dissipation rate a simple binomial conservative (microcanonical) cascade model that reproduces most of the intermittency seen in their observations. Although the viability of this approach rests on scaling symmetry and on the conservation of energy fluxes from large to small scales, a direct connection with the equations of motion is still lacking (Sreenivasan, 1991).

The experimental evidence of multifractality in fully-developed turbulence (e.g. Sreenivasan (1991)), suggests that similar studies could be carried out to understand the intermittent distribution of passive scalars in the lower atmosphere. For this purpose, water vapour scans were measured with the Los Alamos water Raman-lidar at the Campbell Tract of the University of California, Davis. For a more complete description of the field site see Parlange and Katul (1992). These measurements eliminate conventional drift between instruments and provide rapid sampling in both space and time with an accuracy of up to 3\% (Echinger et al., 1994). The measurements were taken continuously for periods of up to a half an hour, over 10 days in August 1991. The accurate and fast-response lidar measurements were carried out over a $500 \mathrm{~m}$ bare soil field with a sampling spatial interval of 1.5 $\mathrm{m}$, and a temporal resolution of up to $8 \mathrm{~Hz}$ for periods of 3-20 min. We refer to this type of data set as a time-dimensional (TD) scan. The validity of idealized assumptions was tested with these scans and it was found that: (i) homogeneity is not valid for distances exceeding $8-10 \mathrm{~m}$; (ii) stationarity holds only in the weak statistical sense; (iii) ergodicity does not hold; and (iv) power-law power spectra are found to scale in the inertial subrange according to Kolmogorov's $-5 / 3$ law [e.g. see Echinger et al., 1993]. Typical power spectra in space and time are shown in Figure 1 together with the $-5 / 3$ line.

In this work we present a multifractal analysis of the water vapour scans.

\section{Review of Multifractal Models}

A stochastic process, $\psi$, (in time or in space) is said to be statistically self-similar if its distribution at a scale $r$ times a factor $\delta$, i.e. of $\psi(r \cdot \delta)$, is the same as the distibution of $\delta^{\gamma} \psi(r)$, for some exponent $\gamma$ and for all $r$ 's and $\delta$ 's. A (discrete) cascade process may account for this geometric behaviour decomposing $\psi$ as a collection of fragments (of possibly different horizontal and vertical sizes) organized 

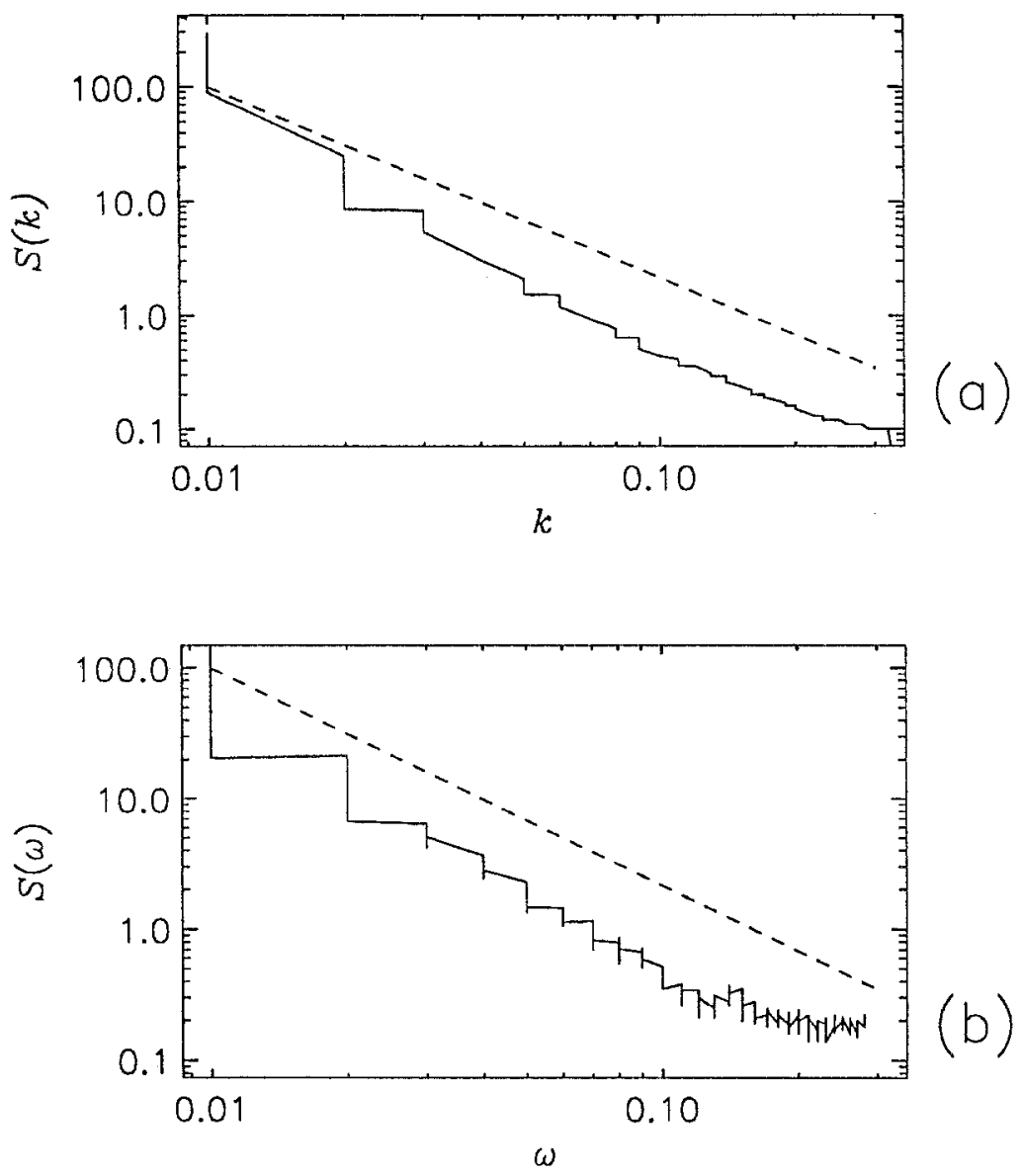

Fig. 1. Space and time power spectra for typical TD scans.

hierarchically following a tree. In the most general case, if the tree has $k$ branches at each node, in the $n$th generation there will be $k^{n}$ fragments with horizontal sizes $l_{1}, l_{2}, \ldots, l_{k^{n}}$. The process may lead to uniform or multifractal representations defined over fractal or non-fractal supports. For example, a fractal object (i.e. like a Cantor set) may appear as the support of a uniform measure when all vertical sizes are the same. A non-uniform (multifractal) measure appears supported by a (possibly) fractal set when the cascade rule gives two or more vertical layers from generation to generation. This general case is relevant since turbulent phenomena exhibit multifractal properties (Sreenivasan, 1991). Extensions of continuous and universal cascades can also be made but will not be considered here; see Wilson et al. (1990) for details.

The nonlinear equation governing the dynamics of turbulent flows has three properties that lead to a cascade phenomenology: (a) scaling symmetry (invariance under zooms); (b) conservation of the energy flux and of scalar dissipation 
(with respective densities $\epsilon$ and $\chi$ ); and (c) transfer of energy across neighboring scales (Kolmogorov's localness in spectral space) (Kolmogorov, 1941). This cascade phenomenology suggests that, even though small-scale fluctuations matter, their structure is intertwined across scales, giving the opportunity to describe the phenomena via a few relevant parameters (Schertzer and Lovejoy et al., 1987).

The most important phenomenological models differ from one to another by their statistical assumptions about the densities $\epsilon$ or $\chi$. Kolmogorov (1941) prompted his investigation of turbulence looking for universal small-scale properties in the flux of energy (or equivalently the energy dissipation rate). Let $L$ be a representative distance of energy containing eddies on a flow travelling at a velocity $u$. Consider the energy dissipation rate, $\epsilon_{r}$ at a scale $r \ll L$, as a statistically stationary field. Then, dimensional arguments predict for the average moment of order $q$ (Kolmogorov, 1941):

$$
\left\langle\left|\frac{(u(x)-u(x+r))^{3}}{r}\right|^{q}\right\rangle=\left\langle\epsilon_{r}^{q}\right\rangle \sim \bar{\epsilon}^{q}
$$

where $\bar{\epsilon}$ is the viscous energy-dissipation per unit mass.

Velocity measurements have shown that Equation (1) is not correct and as a consequence more accurate formulas have been proposed. In agreement with recent developments in multifractal theory, the following has become the virtual standard notation:

$$
\left\langle\epsilon_{r}^{q}\right\rangle \sim \bar{\epsilon}^{q}(r / L)^{D(1-q)-\tau(q)},
$$

where $D$ is the fractal dimension of the support of the data and $\tau(q)$ is the mass exponents function (Halsey et al., 1986).

Intermittency models are basically parameterizations of $\tau(q)$. By virtue of Equation (2), assuming a (convex) form for $\tau(q)$ is equivalent to assuming a probability distribution for $\epsilon_{r}$, i.e., $P\left(\epsilon_{r}\right)$. As will be seen later, Equation (1) corresponds to the concept of a uniform measure over a fractal set (i.e. a monofractal measure) $(\tau(q)=D(q-1))$, while Equation (2) is related to multifractality.

Kolmogorov (1962) modified his original model proposing a lognormal distribution for the energy dissipation probabilities, $P\left(\epsilon_{r}\right)$, so that in Equation (2)

$$
\tau(q)=(1-q)(1-\nu q / 2)
$$

where $\nu$ is an empirical constant known as the intermittency exponent. For the lognormal model this quantity is either $\nu=\tau(2)+1$ or $\nu=\mathrm{d}^{2} \tau(q) / \mathrm{d} q^{2}$. In general however, $\mathrm{d}^{2} \tau(q) / \mathrm{d} q^{2}$ is not a constant, suggesting that the log-normal model is not correct; see Anselmet et al. (1984), Sreenivasan and Kailasnath (1993), Sreenivasan (1991). There are also inconsistencies between three-dimensional cascades and lognormal distributions (Mandelbrot, 1974, 1983) which further disqualify this model; see Schertzer and Lovejoy $(1987,1989)$. 
Historically, the next significant model is the $\beta$ model proposed by Novikov and Stewart (1964); see also Frisch et al. (1978). Here, $\tau(q)$ is a linear function of $q$ :

$$
\tau(q)=(1-\nu)(1-q)
$$

implying that the entire dissipation occurs uniformly on a set of fractal dimension $1-\nu$. This model belongs to the category of uniform fractals. Even though it shares the nonuniformity and intermittency present in data, it gives zero for the support of turbulence, and does not share the observed divergence of moments as noted by Anselmet et al. (1984). This model led however to extensions based on nonuniform fractals (multifractals) which better agree with empirical observations; see Frisch and Parisi (1985).

In the context of multifractals, the $p$-model proposed by Meneveau and Sreenivasan (1987) and the $\alpha$-model proposed by Schertzer and Lovejoy (1983) are among the best known phenomenological models. The $p$-model corresponds to:

$$
\tau(q)=\log _{2}\left(p^{q}+(1-p)^{q}\right)
$$

while the $\alpha$-model has:

$$
\begin{aligned}
\tau(q)= & \log _{2}\left[2 \alpha\left(1 / 2-\sqrt{(1-\alpha) \frac{2^{\nu}-1}{4 \alpha}}\right)^{q}+2(1-\alpha)\right. \\
& \left.\times\left(1 / 2+\sqrt{\alpha \frac{2^{\nu}-1}{4(1-\alpha)}}\right)^{q}\right] .
\end{aligned}
$$

This last formula is chosen such that $\tau\left(q_{c}\right)=0$ for some critical value, $q_{c}>1$, and $1-2^{-\nu}<\alpha<1$; see Schertzer and Lovejoy (1987) and Borgas (1992). In the limit when $\alpha \rightarrow 1 / 2$, Equation (6) tends to Equation (5) if $p=1 / 2+\sqrt{2^{\nu}-1}$. In this sense, the $\alpha$-model could be considered as an extension of the $p$-model but the two models arise from different kinds of cascades. The $p$-model comes from a simple cascade that conserves mass on each step of the construction, i.e. a microcanonical cascade. The $\alpha$-model arises from a canonical cascade, as it does not preserve mass exactly all the way but rather does it on the average. These two models offer the best estimates for the measured intermittency exponent (evaluated from second-order derivatives of $\tau(q)$ at $q=0$ ). The $p$-model fit is best for the energy dissipation rate $\epsilon$ when $p=0.7$ (Meneveau and Sreenivasan, 1987), while the $\alpha$-model yields $\nu=1+\tau(2)=0.214$ for different values of $\alpha$, e.g. $\alpha=0.927$ when $q_{c}=10$ or $\alpha=0.9$ when $q_{c}=17$ (Borgas, 1992).

Another phenomenological model which arises from canonical cascades leading to Lévy stable processes is the universal multifractals model of Schertzer and Lovejoy (1987). For this model the mass exponents give

$$
\tau(q)= \begin{cases}H q+C_{1}\left(q^{\beta}-q\right) /(\beta-1)+1-q & \beta \neq 1 \\ H q+C_{1} q \log (q)+1-q & \beta=1,\end{cases}
$$


where $H$ defines how canonical is the cascade, i.e. its deviation from conservation, $C_{1}$ is the codimension of the set of mean singularities, and $\beta$ is the Lévy index of the process. This model has been found useful for describing the shape of $\tau(q)$ for several geophysical phenomena; see Gabriel et al. (1988), Lovejoy and Schertzer (1990a), Lavallée et al. (1991a), and Tessier et al. (1993a, 1993b).

As stated earlier, the intermittency exponent is not uniquely defined. However, in analogy to the heat capacity in thermodynamics, one can define the notion of the intermittency function, $\nu(q)=\mathrm{d}^{2} \tau(q) / \mathrm{d} q^{2}$ to aid in the selection of a model for actual data. Figure 2 shows the different kinds of functions that appear when using alternative models. As may be seen, for the $p$-model, $p \neq 0.5, \nu(q)$ has a bell shape that peaks at the origin, the $\alpha$-model (for parameters which closely fit fully-developed turbulence) possesses one peak but it is not necessarily located at the origin, while the universal multifractals model decays for increasing $q$ 's but diverges at the origin. The intermittency function will be computed when analyzing water vapour data.

Multifractal behaviour is usually summarized by the spectrum of singularities of the data set under study. The typical assumption is to interpret such data as a measure defined over the relevant support, either in time or in space. Several characteristics of such a spectrum are used to quantify the data. These include the entropy dimension, the correlation dimension and (when $q=2$ ) the previously mentioned intermittency exponent. The basic multifractal formalism is briefly reviewed next. For further details the reader is referred to Feder (1988) and Mandelbrot (1989).

Suppose that the effective length $L$ of a spatial (or temporal) positive series can be divided into $N(r)$ cells, each of length $r$. Then, it is possible to represent such series as if it were a (normalized) probability mass function $(\mu)$, assigning at the $i$ th cell the quantity $\mu_{i}=\chi_{i} / \chi$, where $\chi_{i}$ is the measure over the $i$ th subinterval of size $r$, and $\chi$ is the total measure. According to the formalism, the intermittency of the measure $\mu$ is characterized by the statistically self-similar behaviour of the fractional mass at the $i$ th cell, $\mu_{i}(r)$, at resolution $r$,

$$
\mu_{i}(r) \sim r^{\alpha}
$$

where $\alpha$ controls the local singularity of the measure, and is known as the LipschitzHölder exponent. The data set is termed multifractal, if the number of cells characterized by the exponent $\alpha$ at resolution $r, N(r, \alpha)$, satisfies

$$
N(r, \alpha) \sim r^{-f(\alpha)}
$$

where $f(\alpha)$ is interpreted (when possible) as the fractal dimension of the cells whose local mass have a local singularitiy $\alpha$. For each $\alpha$ there corresponds a single value of $f(\alpha)$ and the graph $f$ vs. $\alpha$ (typically an inverted parabola) represents the measure's multifractal spectrum.

An alternative way for arriving at the concept of multifractality has been developed by Schertzer and Lovejoy (1987). Instead of working with the measure $\mu(r)$, 

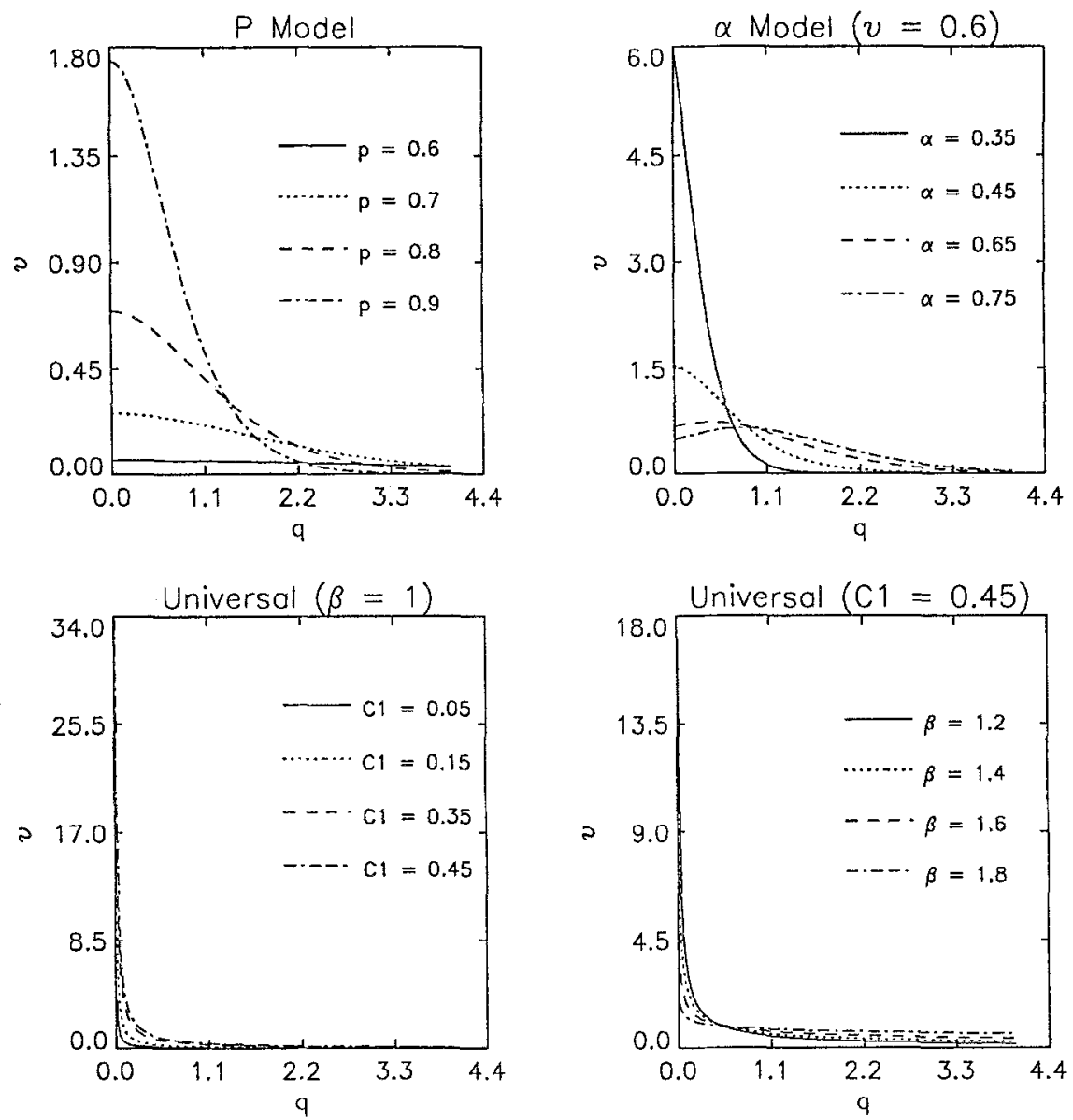

Fig. 2. Intermittency function for the $p$-model $(p=0.6,0.7,0.8,0.9)$, the $\alpha$-model $(\nu=0.6, \alpha=$ $0.35,0.45,0.65,0.75)$, and the universal model $\left(H=0, \beta=1, C_{1}=0.05,0.15,0.35,0.45\right.$, and $C_{1}=$ $0.45, \beta=1.2,1.4,1.6,1.8)$.

attention is centered on the extremes of the density $\epsilon_{\lambda}=\mu(r) / r$. This leads to the codimension function of the series, $c(\gamma)$, via the scaling relation

$$
\operatorname{Prob}\left[\epsilon_{\lambda} \geq \lambda^{\gamma}\right] \sim \lambda^{-c(\gamma)} .
$$

The two multifractal formalisms are identical only when large extremes of the data (series) are considered. The codimension function is related to the left side of the multifractal spectrum via the relations: $\alpha=1-\gamma$ and $f(\alpha)=1-\gamma$. For data sets defined over two or three dimensions, the codimension function is preferred because it does not depend explicitly on the dimension being considered (Schertzer and Lovejoy, 1989).

A measure's multifractal spectrum (or codimension function) may be computed following alternative methods. The most common procedures for a multifractal 
spectrum are the method of moments (or partition function) (Frisch and Parisi, 1985) and the direct method (Chhabra and Jensen, 1989). For the codimension function, there are also several procedures. They include the Probabilistic Distribution Multiple Scaling (PDMS) technique (Schertzer and Lovejoy, 1989), and the Double Trace Moment procedure of Lavallee et al. (1991b). We shall concentrate only on the methods for a multifractal spectrum as all procedures give, in lieu of the existing equivalences, identical results.

The method of moments exploits the scaling property of the $q$-th moment of $\mu_{i}(r)$ :

$$
M(q, r)=\sum_{i=1}^{N(r)}\left(\mu_{i}(r)\right)^{q} \sim(r)^{-\tau(q)}
$$

which involves the aforementioned mass exponents of order $q \tau(q)$. Observe that $\tau(q)$ plays the role of a fractal dimension for a fixed $q$. When $q=0, \tau(0)$ gives the fractal dimension of the support of the measure, which for the data in this work gives 1 . When $q=1$, the sum in (11) gives $1\left(=r^{0}\right)$ for all $r$, and therefore $\tau(1)=$ 0 .

From its definition, it is clear that the exponent $\tau(q)$ is obtained from the slopes of $\log -\log$ plots of $M(q, r)$ vs. $r$. Following Frisch and Parisi (1985) one can relate the exponents $\tau(q)$ with $\alpha$ and $f(\alpha)$ via Legendre transformations:

$$
\begin{aligned}
& f(\alpha(q))=q \alpha(q)+\tau(q) \\
& \alpha(q)=-\frac{\mathrm{d} \tau(q)}{\mathrm{d} q} .
\end{aligned}
$$

The method of moments consists of finding the mass exponents first and then obtaining $\alpha$ and $f(\alpha)$ via Equations (12) and (13). The direct method does not rely (numerically) on the Legendre transforms, but rather uses the thermodynamic analogy of $\alpha$ and $f(\alpha)$ being related to the internal energy and entropy of the system, respectively. This leads to the following implicit functions on the "inverse temperature" $q$ :

$$
\begin{aligned}
& f(q)=\lim _{r \rightarrow 0} \frac{\sum_{i}^{N} \mu_{i}(q, r) \log \left(\mu_{i}(q, r)\right)}{\log (r)} \\
& \alpha(q)=\lim _{r \rightarrow 0} \frac{\sum_{i}^{N} \mu_{i}(q, r) \log \left(\mu_{i}(r)\right)}{\log (r)}
\end{aligned}
$$

where $\mu_{i}(q, r)=\mu_{i}(r)^{q} / M(q, r)$. Chhabra and Jensen (1989) established that this method works well for turbulence and for dynamical systems. Observe that in essence both multifractal spectrum methods are based on the same thermodynamic 
relations, and hence give very close results. The direct method just avoids errors when finding Legendre transformations numerically.

The thermodynamic relations underline the importance of the entropy dimension of the measure defined by $\alpha_{1}=\alpha(q=1)=f(\alpha(q=1))$. Its name stems from the observation that, using (15), $\alpha_{1} \log (r) \sim \sum_{i} \mu_{i} \log \left(\mu_{i}\right)$ which is the thermodynamic entropy. For one-dimensional series, $\alpha_{1}$ takes values between 0 and 1 . A value of 1 signifies an evenly spread distribution of irregularities across the length of the series, i.e. a measure with no singularities at all or a continuous distribution (e.g. a uniform or a normal). When $\alpha_{1}<1$, there exists a subset of the support of the measure, whose fractal dimension is $\alpha_{1}$, when the irregularities concentrate (Feder, 1988). Therefore, this parameter quantifies the degree of "fuzziness" or "emptiness" of the irregularities present in the data set. Notice that in relation to the universal multifractal model of Schertzer and Lovejoy (1987), $C_{1}=D-\alpha_{1}$, with $D$ being the dimension of the support of the data.

Another useful parameter, due to its importance in phase-space representations of non-linear dynamical systems, is the correlation dimension defined by $D_{2}=$ $2 \alpha(2)-f(\alpha(2))$. This qualifier receives its name because the two-point correlation function, $c(r)$, scales as $r^{D_{2}}$ (McCauley, 1990).

A cautionary note should be made when interpreting computed $f(\alpha)$ values. There exist some experimental instances for which $f(\alpha)$ can take on negative values. In such cases, $f(\alpha)$ can not be interpreted as a fractal dimension. Mandelbrot $(1990,1991)$ suggests a stochastic interpretation which has become the subject of recent discussions (Lovejoy and Schertzer, 1990b; Chhabra and Sreenivasan, 1991a, 1991b; Puente et al., 1995). This new interpretation has given further life to the philosophical debate of determinism vs. randomness in natural phenomena. As our data resulted in negative dimensions in only a few scans, and as this issue is still not completely understood, we shall not dwell on it.

\section{Experiments}

The experimental data used in this analysis were collected at the Campbell Tract at the University of California, Davis in August, 1991. Water vapour measurements were gathered over a bare soil field which extends about 400 by $400 \mathrm{~m}$. The solar-blind water-Raman lidar used in this experiment is based upon the technique pioneered by Cooney et al. (1985). A pulse of high-energy laser light is emitted into the atmosphere by the instrument, and its 'echo' (nitrogen and water-vapour signals) is detected some time later. By scanning the instrument, the surface profile may then be deduced from the continuous stream of pulses because the position of the platform is accurately known. Figure 3 illustrates the location of the water-Raman lidar, the accessory instruments used to make the measurements and the region where the meteorological data were taken. All of the meteorological instruments were located at ranges in which there is confidence in the lidar's accuracy. 


\section{EXPERIMENTAL SETUP}

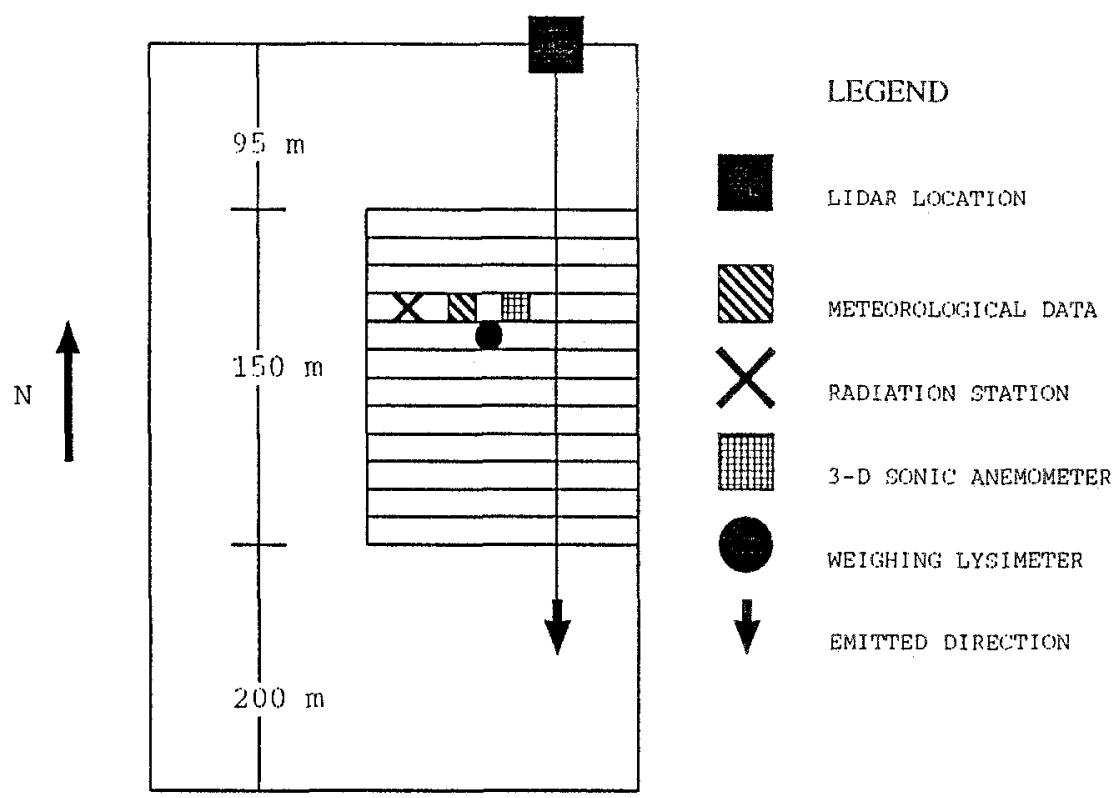

Fig. 3. Location of Raman-lidar and other instruments.

Each pulse is normalized by dividing the water vapour return signal coming from the lidar by that coming from nitrogen. The normalized return signals are directly proportional to the absolute water-vapour content of the air and require only a correction for differential ozone absorption at the two return wavelengths. The lidar has been demonstrated to duplicate the measurements of conventional instruments to within 3-5\% providing high accuracy and long confidence ranges for our experiment. For further details, the reader is referred to Eichinger $e t$ al. (1994).

The observations used in our analysis were sampled at fixed points in space under various atmospheric stability conditions. The Obukhov length was computed to summarize these conditions. Table I shows the atmospheric conditions above the drying land surface where the lidar measurements were taken. Our analysis included stable, unstable and neutral conditions.

The data set was taken by firing the laser along a single line-of-sight parallel to the ground, oriented opposite to the mean wind direction. A given number of laser pulses was summed, saved to the disk, and the process was repeated until the available computer disk space was filled. Each series of one-dimensional scans taken in this manner creates a two-dimensional array. Each time-dimensional scan, $\operatorname{TD}(i, j)$, has elements which represent the water vapour concentration at time $i$-th (s) and at the $j$-th location $(\mathrm{m})$. Since the number of laser pulses that were 
TABLE I

Atmospheric conditions above the drying land surface

\begin{tabular}{|c|c|c|c|c|c|c|c|c|c|}
\hline \multirow[t]{3}{*}{ Scan } & \multirow{2}{*}{\multicolumn{2}{|c|}{$\begin{array}{l}\text { Date } \\
\text { Aug/91 }\end{array}$}} & \multicolumn{2}{|l|}{ Wind } & \multirow[t]{3}{*}{ Stability } & \multirow{2}{*}{\multicolumn{2}{|c|}{$\frac{\text { Raw }}{\text { TD array }}$}} & \multirow{2}{*}{\multicolumn{2}{|c|}{$\frac{\text { Taylor's }}{\text { TD array }}$}} \\
\hline & & & Azimuth & Speed at $2 \mathrm{~m}$ & & & & & \\
\hline & Day & Hour & Degrees $N$ & $\mathrm{~m} / \mathrm{s}$ & & Row & Col. & Row & Col. \\
\hline 1 & 24 & $19: 01$ & 164 & 1.25 & Stable & 400 & 100 & 400 & 100 \\
\hline 2 & 24 & $17: 31$ & 195 & 6.46 & Unstable & 500 & 100 & 500 & 40 \\
\hline 3 & 24 & $17: 44$ & 198 & 6.50 & Unstable & 500 & 100 & 500 & 42 \\
\hline 4 & 23 & $21: 04$ & 189 & 5.80 & Neutral & 500 & 100 & 500 & 48 \\
\hline
\end{tabular}

summed varied from 5 to 100 , the rate at which data collection frequencies changed ranged from $0.1-2.0 \mathrm{~s}$, giving 3-20 min for the total length of the time series. The dimensions of the TD arrays, considering only those measurements deemed accurate for each scan, are also shown in Table I. Bearing in mind that tests of Taylor's hypothesis will be reported later, the spatial data are suitably transformed by the appropriate wind velocity to give the vector sizes reported on the last column of Table I. As may be seen, this adjustment clearly reduces the number of available data in space for the scans 2, 3 and 4 .

Since many scans and relatively long distances were involved in the experiment, we have an adequate and accurate data set of about one-half million points for a multifractal analysis. However, we must be careful in generalizing the conclusions we obtain when testing Taylor's hypothesis due to the reduction of available data in space when spatial scales are dimensionalized by the wind velocity factor. The multifractal analysis was carried out for a quantity which mimics the scalar dissipation rate of the water vapour content for each scan. We defined a scalar gradient measure as

$$
\chi=\left(\partial \theta / \partial x_{i}\right)^{2} \quad i=1,2
$$

where $x_{1}$ and $x_{2}$ represent spatial and temporal coordinates respectively, and $\theta$ is the passive scalar (the water vapour). In our analysis, we calculated "temporal dissipation rates" for fixed times, $t_{0}$, by computing their partial derivatives with respect to distance $x$ :

$$
\chi_{x}=\left\{\frac{\partial \theta\left(x, t_{0}\right)}{\partial x}\right\}^{2} .
$$

Similarly, "spatial dissipation rates" were computed for fixed locations, $x_{0}$, via partial derivatives with respect to time $t$ :

$$
\chi_{t}=\left\{\frac{\partial \theta\left(x_{0}, t\right)}{\partial t}\right\}^{2} .
$$


Notice that $\chi_{t}$ is much closer to the actual dissipation rate than $\chi_{x}$.

These two measures were calculated numerically at two different scales, $r$ and $2 r$ where $r$ is the minumum distance between measurements either in time or space. Dissipation values at resolution $2 r$ were computed by means of two alternative methods, which were named the deriv1 and the deriv 2 procedure. In the deriv1 method, all $N(r) / 2$ pairs of consecutive local water vapour contents at resolution $r$ are summed to obtain a new water vapour measure at the $2 r$ scale, $\theta(2 r)$, and then the scalar dissipation rate is computed from its definition, i.e. Equation (16). In the deriv 2 procedure, dissipations at the second scale are obtained directly from the scalar dissipation rate at resolution $r$, simply by adding all consecutive rates by pairs. In both cases, each one-dimensional measure was normalized by the total mass in the respective one-dimensional cut.

Notice that the two procedures are not related to one another, deriv 2 relies on the idea of a standard additive cascade for the field of dissipation. This gives a linear transition from one scale to the next. On the other hand, by virtue of squaring the derivatives of the passive scalar, deriv 1 results in a non-linear definition of dissipation rates, which can not be obtained from previous resolutions just by adding. As will be seen later, different multifractal properties are obtained when using the alternative definitions.

A comparison of the different scans may be made by considering jointly all the two-dimensional (space-time) water vapour measurements. This allows the definition of measures over time-space $\hat{\chi}_{t}$ and $\hat{\chi}_{x}$ which correspond to the onedimensional measures $\chi_{t}$ and $\chi_{x}$. They included also raw data or data transformed (in space) to account for Taylor's hypothesis. The multifractal analysis presented in this work is based on the method of moments, as no substantial differences were found when using the direct method.

\section{Results and Discussion}

Substantial temporal and spatial variability was observed for the water vapour content measurements, $\theta$, for all the scans. Figure 4 shows the typical variability exhibited by the water vapour content on the four TD scans analyzed. Despite the notorious variability in $\theta$, a cascade phenomenology did not explain such behaviour.

As previously explained, we focused our multifractal analysis on the "scalar dissipation rate", $\chi$, computed for each possible spatial and temporal section, i.e. for all plausible samples of $\chi_{t}$ and $\gamma_{x}$. Both rates were computed via the deriv1 and deriv 2 procedures. For each procedure the water vapour density was calculated according to each of the last two columns in Table I, i.e. considering the raw data or transforming the space coordinates according to average wind velocities. Figure 5 illustrates the typical behaviour of $\chi_{t}$ and $\chi_{x}$ ((a) and (b) respectively) when raw data are used and the dissipation rates are found from the deriv 1 and 

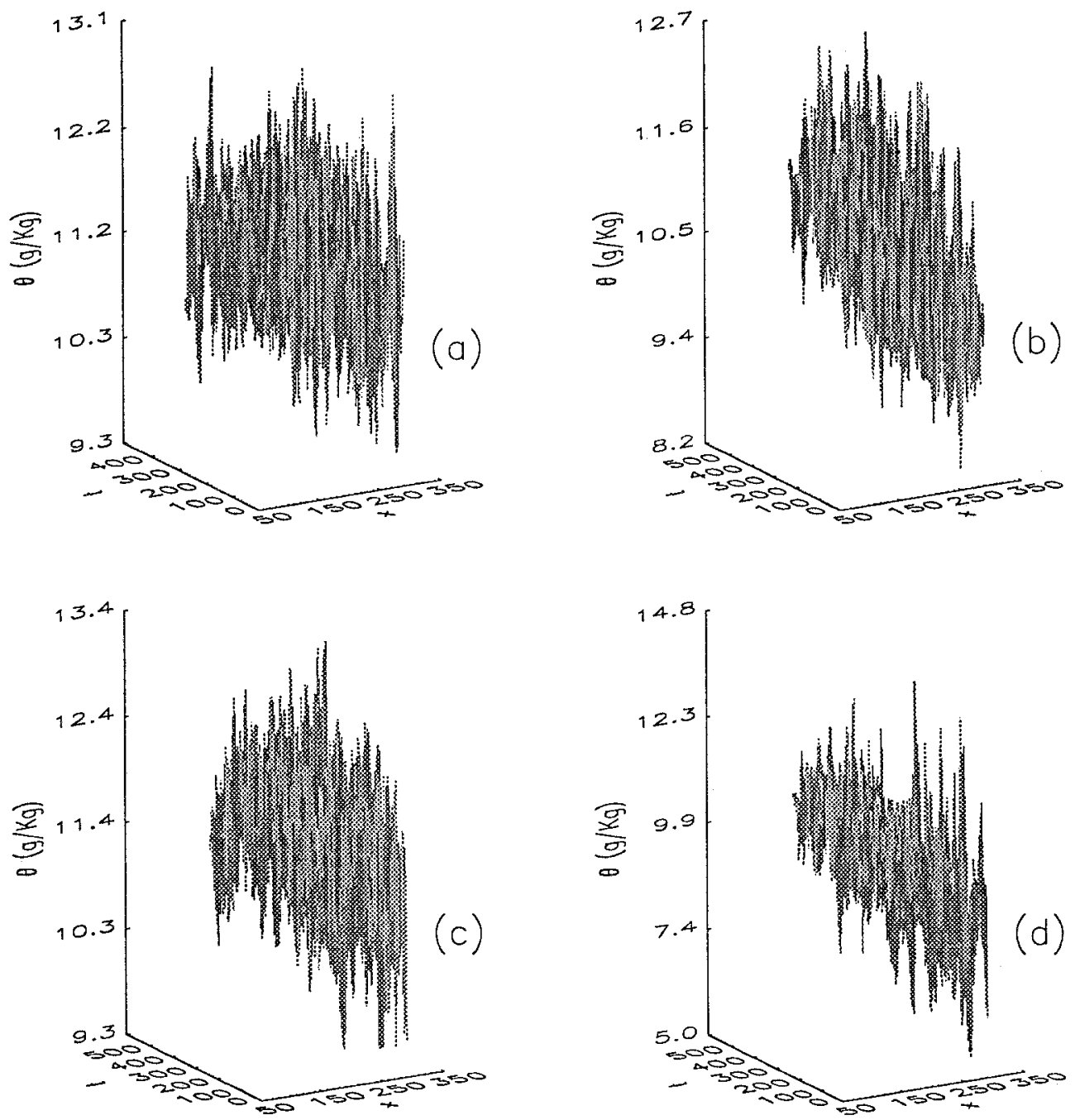

Fig. 4. Lidar measured water vapour content of scans: (a) 1, (b) 2, (c) 3, and (d) 4 .

deriv2 procedures (on the first and second columns, respectively). The particular observations of this figure correspond to scan 2 at location $x_{0}=119 \mathrm{~m}$ from the lidar and at $t_{0}=2 \mathrm{~s}$.

Typical behaviour of $\chi_{t}$ and $\chi_{x}$, when data in space are transformed according to wind velocities and averaging is performed according to the last column in Table $\mathrm{I}$, is illustrated in Figure 6 . Notice that in comparison to Figure 5 less observations are available. Observe also that the graphs in part (b) of Figures 5 and 6 exhibit a higher degree of variation than those in part (a).

The multifractal spectrum for each possible one-dimensional cut of $\chi$ (i.e. $\chi_{t}$ and $\chi_{x}$ ) was calculated by means of the method of moments (Frisch and Parisi, 

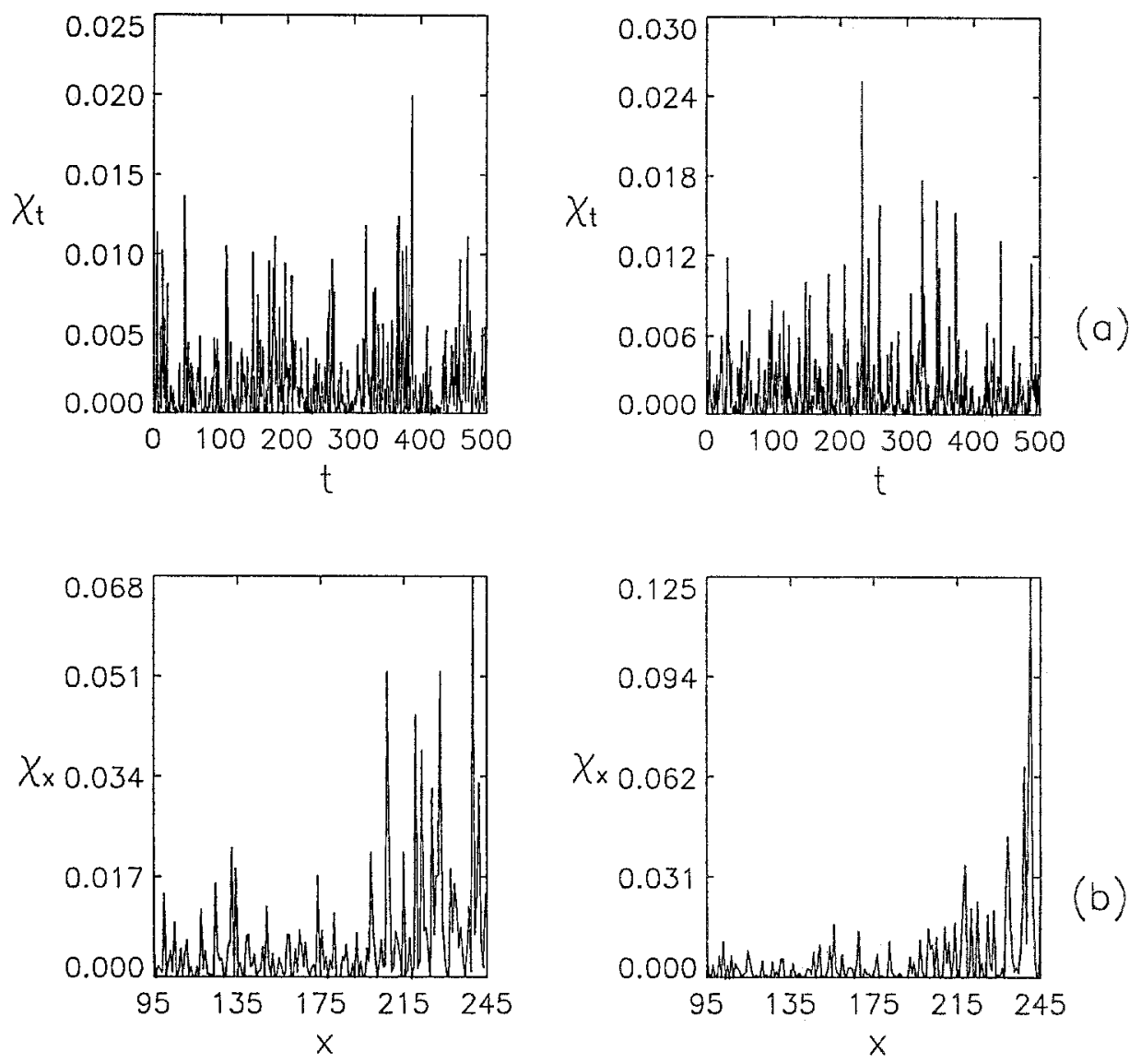

Fig. 5. One-dimensional section for (a) $\chi_{t}$ at $x_{0}=119 \mathrm{~m}$ from lidar, and for (b) $\chi_{x}$ at $t_{0}=2 \mathrm{~s}$, under deriv 1 (left) and deriv 2 (right) procedures for dissipation calculations. Raw data are used from scan 2.

1985). Figures 7 and 8 show the average multifractal spectra obtained for the four different combinations considered in Figures 5 and 6, respectively. These graphs include information not only for scan 2 but also for the other scans. In accordance with the work of Schertzer and Lovejoy (1987) only the large values of the measures appear on the left branch of the multifractal spectra.

As may be seen, all plots in Figure 7 are almost identical to those in Figure 8 indicating that average multifractal spectra from raw or transformed data match. It is also noticeable that similarity exists among the multifractal spectra of all the scans under all alternative atmospheric conditions. This implies that for the "water vapour dissipation": (a) Taylor's hypothesis appears to hold, and (b) prevailing atmospheric stability conditions appear to be unimportant.

Here, a cautionary note must be made when comparing parameters of one intermittency model to another. Description of turbulence requires a proper definition 

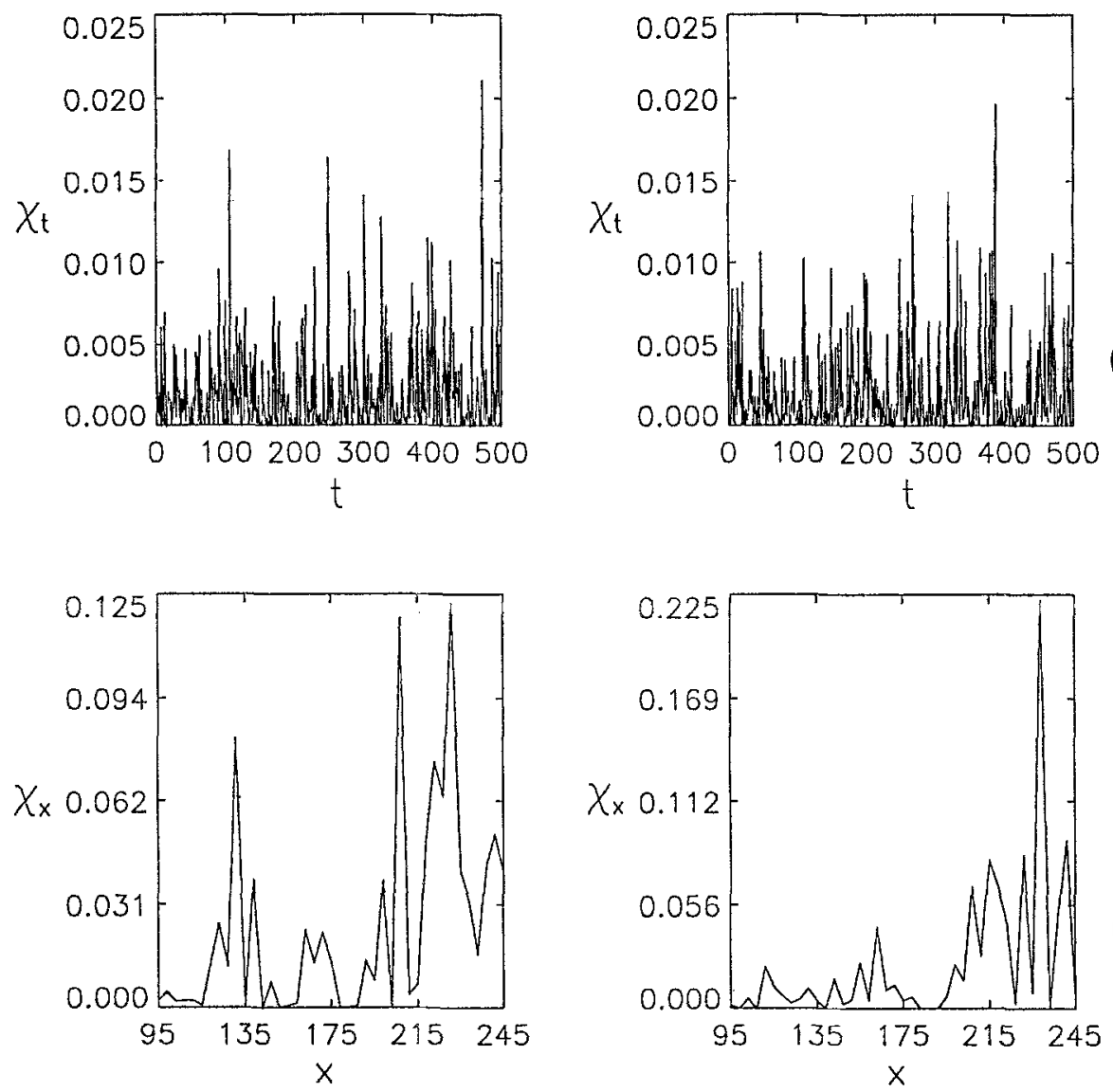

(b)

Fig. 6. One-dimensional section for (a) $\chi_{t}$ and $x_{0}=119 \mathrm{~m}$ from lidar, and for (b) $\chi_{x}$ at $t=2 \mathrm{~s}$, under deriv 1 (left) and deriv2 (right) procedures for dissipation calculations. Data in space are transformed according to wind velocities and were taken from scan 2.

of the measure that is being analyzed, because in essence, the method used to calculate dissipation rates dictates the most relevant parameters used in intermittency models. For comparison purposes, and bearing in mind that alternative spectra are obtained when looking at $\chi_{x}$ and $\chi_{t}$, Tables II and III include averages and standard deviations of the intermittency exponent $\nu$, the entropy dimension $\alpha_{1}$ and the correlation dimension $D_{2}$, for raw data and dissipation computed via the deriv 1 and deriv 2 procedures, respectively. Observe that markedly different multifractal spectra are obtained from the alternative method of dissipation calculations, as seen comparing the curvatures of $f(\alpha)$ curves on the left (deriv1) and right (deriv2) columns of Figures 7 and 8 . The deriv 2 measures result in lower entropies $\alpha_{1}$, and hence have more intermittency than those obtained via the deriv1 procedure.

Tables II and III show that there are indeed significant differences between the reported multifractal parameters. Consistently, the deriv 2 procedure gives measures 

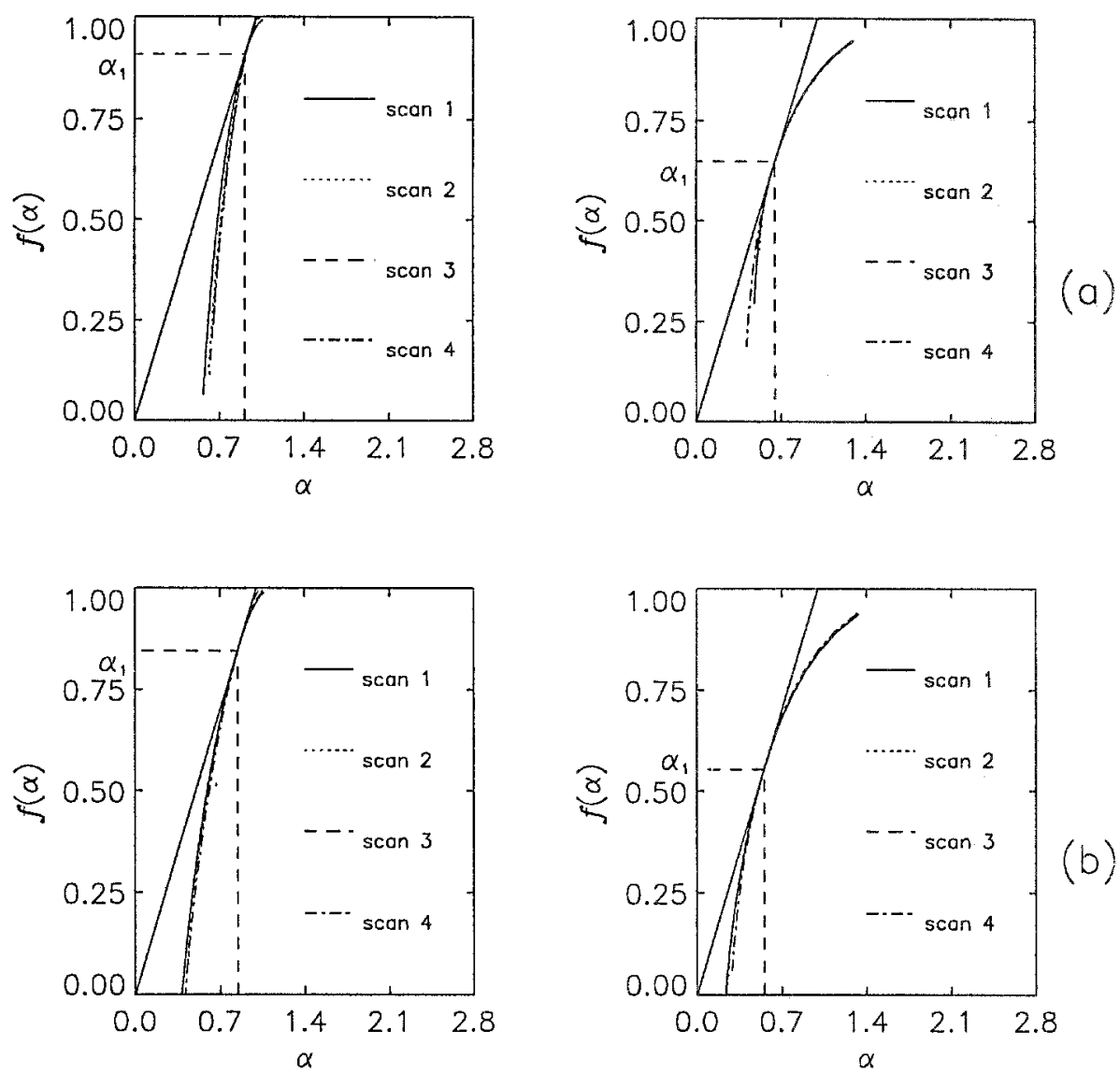

Fig. 7. Average one-dimensional multifractal spectra for deriv 1 (left) and deriv2 (right) dissipations. (a) $\chi_{t}$, and (b) $\chi_{x}$. Raw data are used and averaging is performed according to the second to last column in Table I.

which are more intermittent than those obtained by deriv 1 dissipation calculations, as exemplified by a higher intermittency exponent $(\nu)$, lower entropy $\left(\alpha_{1}\right)$ and a lower correlation dimension $\left(D_{2}\right)$. This means that by considering a "geometric" averaging of dissipation values results in data that look fuzzier or emptier than when actual water vapour data are used to find the dissipation rate at the coarse scale. Even so, mathematically speaking, deriv1 appears to be more correct as it captures the non-linear variability in the underlying data, deriv 2 is more consistent with the multifractal formalism. This is illustrated in Figure 9 in relation to mass exponents given by both methods. Notice that deriv 2 gives a convex mass exponent curve, while deriv 1 does not for negative values of the exponent $q$.

Tables II and III also show that the dissipation rates for fixed times, $\lambda_{x}$, are consistently more intermittent than those at fixed locations $\chi_{t}$. This result appears independently of using either raw or transformed data. This reveals that the scalar 

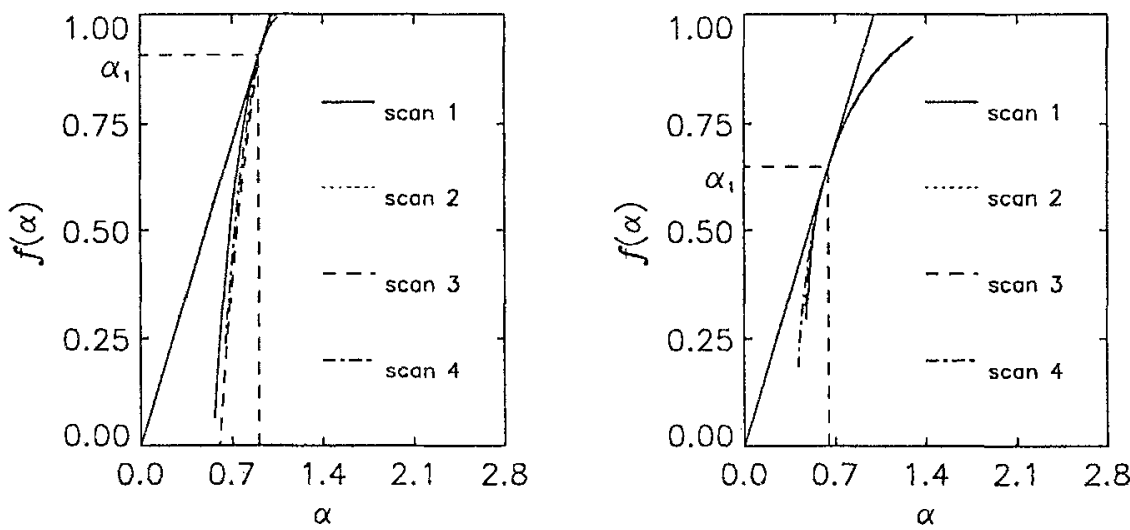

(a)
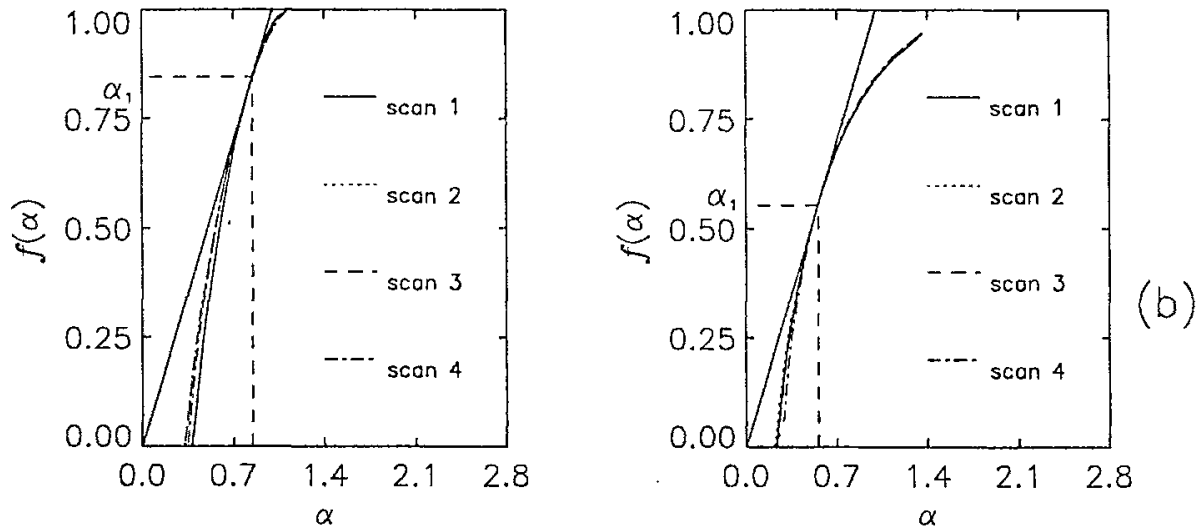

Fig. 8. Average one-dimensional multifractal spectra for deriv 1 (left) and deriv2 (right) dissipations. (a) $\chi_{t}$, and (b) $\chi_{x}$. Data in space are transformed according to wind velocities and averaging is performed according to the last column in Table I.

TABLE II

Multifractal spectra parameters for one-dimensional deriv I dissipation measures and raw data

\begin{tabular}{lllllll}
\hline \multirow{2}{*}{ Scan } & \multicolumn{1}{l}{$\gamma_{t}$} & \multicolumn{1}{l}{$\alpha_{1}$} & $D_{2}$ & $\chi_{x}$ & $\alpha_{1}$ & $D_{2}$ \\
\hline 1 & $0.43 \pm 0.002$ & $0.90 \pm 0.008$ & $0.84 \pm 0.003$ & $0.91 \pm 0.003$ & $0.84 \pm 0.005$ & $0.68 \pm 0.001$ \\
2 & $0.17 \pm 0.008$ & $0.95 \pm 0.001$ & $0.88 \pm 0.004$ & $0.61 \pm 0.004$ & $0.86 \pm 0.006$ & $0.68 \pm 0.001$ \\
3 & $0.11 \pm 0.004$ & $0.96 \pm 0.001$ & $0.90 \pm 0.004$ & $0.68 \pm 0.004$ & $0.85 \pm 0.006$ & $0.68 \pm 0.002$ \\
4 & $0.08 \pm 0.002$ & $0.96 \pm 0.001$ & $0.90 \pm 0.006$ & $0.47 \pm 0.007$ & $0.90 \pm 0.005$ & $0.72 \pm 0.002$ \\
\hline
\end{tabular}



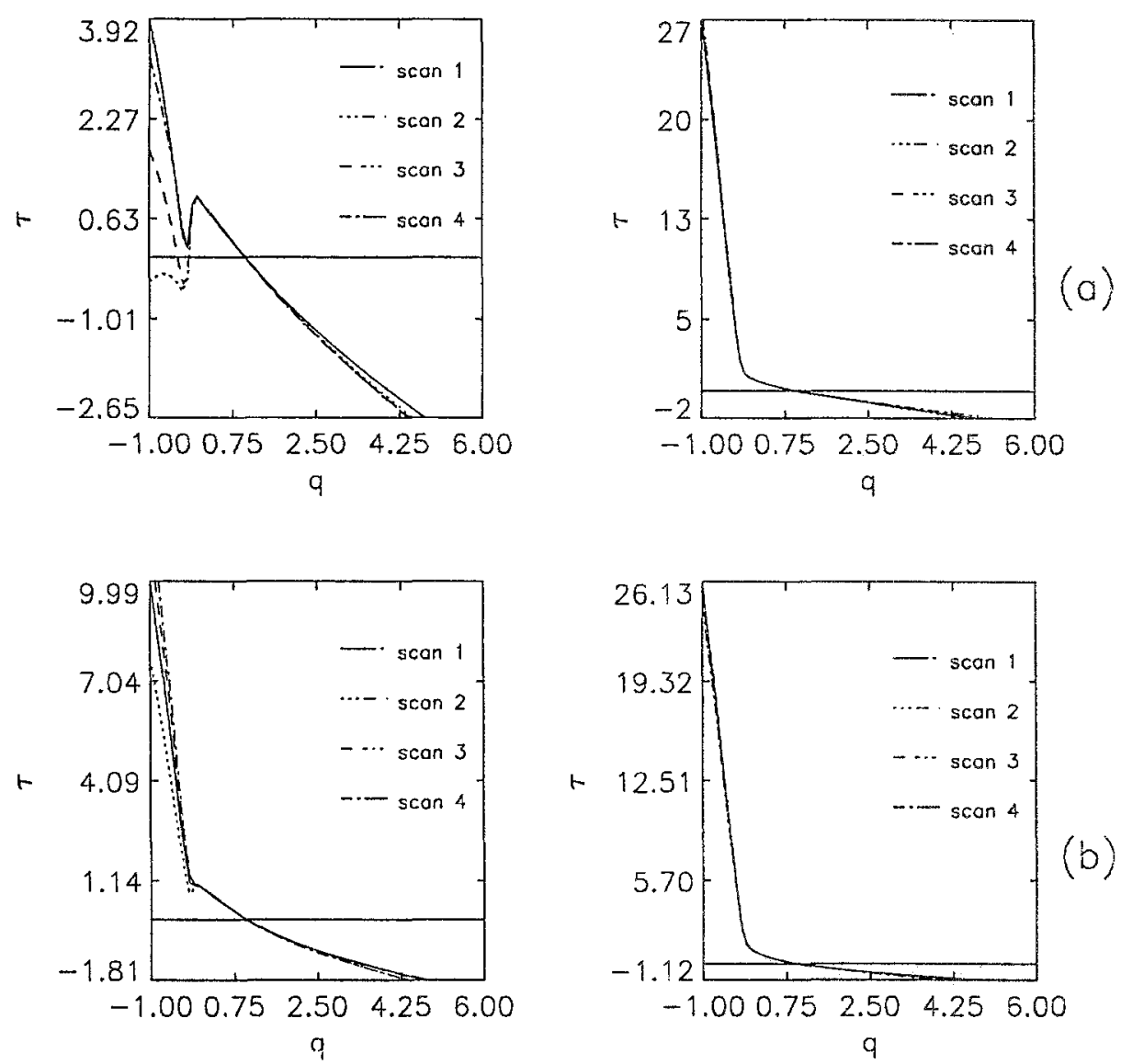

Fig. 9. Average $\tau(q)$ vs. $q$ for one-dimensional deriv 1 (left) and deriv2 (right) dissipations. (a) $\backslash t$, and (b) $\chi_{x}$. Raw data are used and averaging is performed according to the second to last column in Table I.

TABLE III

Multifractal spectra parameters for one-dimensional deriv2 dissipation measures and raw data

\begin{tabular}{lllllll}
\hline \multirow{2}{*}{ Scan } & \multicolumn{7}{l}{$x_{t}$} & \multicolumn{6}{l}{$x_{x}$} & & \\
\cline { 2 - 7 } & $\nu$ & $\alpha_{1}$ & $D_{2}$ & $\alpha_{1}$ & $\alpha_{1}$ & $D_{2}$ \\
\hline 1 & $3.33 \pm 0.005$ & $0.65 \pm 0.001$ & $0.58 \pm 0.001$ & $4.31 \pm 0.006$ & $0.55 \pm 0.005$ & $0.41 \pm 0.002$ \\
2 & $3.35 \pm 0.002$ & $0.65 \pm 0.001$ & $0.58 \pm 0.005$ & $4.16 \pm 0.003$ & $0.56 \pm 0.003$ & $0.42 \pm 0.001$ \\
3 & $3.33 \pm 0.003$ & $0.65 \pm 0.002$ & $0.57 \pm 0.003$ & $4.13 \pm 0.001$ & $0.55 \pm 0.003$ & $0.41 \pm 0.001$ \\
4 & $3.28 \pm 0.003$ & $0.64 \pm 0.003$ & $0.55 \pm 0.002$ & $3.92 \pm 0.005$ & $0.58 \pm 0.001$ & $0.45 \pm 0.002$ \\
\hline
\end{tabular}


dissipation between neighboring spatial scales looks "emptier" than those between neighboring temporal scales. This observation does not contradict Taylor's hypothesis. The multifractal spectrum is a local description of interwoven events that occur across neighboring spatial or temporal scales (cascade phenomenology) while Taylor's hypothesis is a global characteristic of the process. As previously seen, average multifractal spectra from raw or transformed data resulted indeed in similar behaviour. Even though there are more data available in time than in space, the disparity in intermittency between space and time is not just numerical. In fact, when scans in time are sampled so that their size match those in space, the results are still quite similar to those reported in time for $\chi_{x}$.

As previously mentioned when describing averaged multifractal spectra, there are few differences when comparing relevant parameters from different scans; see Tables II and III. This indicates that for the data sets considered, stability conditions are not relevant if a description of interwoven events is made.

It is worth noticing that in relation to the scalar dissipation rates reported in Sreenivasan et al. (1989) ( $\nu=0.36 \pm 0.05)$, there are no scans which give such behaviour. Instead, all sets considered produce "apparent" intermittency exponents which are much larger, especially when using the deriv 2 procedure.

In an attempt to discover which is the proper structure for a model of the observed intermittency, we analyzed the natural extension of the intermittency exponent to alternative weights $q, \nu(q)=\mathrm{d}^{2} \tau(q) / \mathrm{d} q^{2}$. This function was computed for raw and transformed water vapour data (Figures 10 and 11, respectively), employing both the deriv 1 and deriv 2 procedures for dissipation calculations and for all available scans. As can be seen, $\nu(q)$ exhibits a non-symmetric (gamma-like) behaviour plus a singularity at the origin, when the deriv 1 method is used. This is found for both $\chi_{t}$ and $\chi_{x}$ and suggests that none of the three popular models included in Figure 2 may fit such behaviour. On the other hand, the observed decay given by deriv 2 may be nicely captured if the universal multifractal model is used; compare Figures 10 and 11 with Figure 2. Notice that the intermittency exponent at the origin appears finite for all scans, but given the resolution of the data a plausible singularity can not be discarded.

A multifractal analysis was also carried out considering jointly all the bidimensional water vapour measurements. Figure 12 presents $\nu(q)$ for such bidimensional dissipation measures computed via the deriv 2 procedure (computing coarse scales over time-space rectangles). When the deriv 1 procedure was used, multifractality was not detected. As may be seen, results are consistent with the predicted shape of the universal multifractal model, despite oscillations seen on scan number 4 . This suggests that such a model, with only three parameters, may capture the intermittency of the two-dimensional measures. In regards to the most important intermittency parameters, Table IV shows that dissipations in space $\left(\hat{\chi}_{t}\right)$ lead to less intermittency than those in time $\left(\hat{\chi}_{s}\right)$. Observe that these were precisely the trends found with the one-dimensional scans. Notice the close agreement found in all parameters when using alternative scans. 

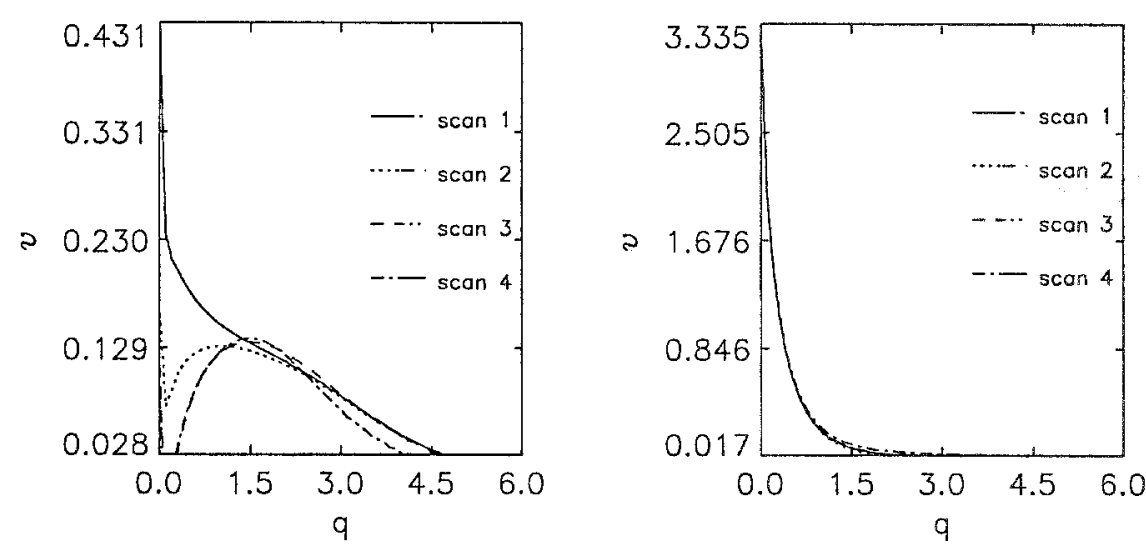

(a)
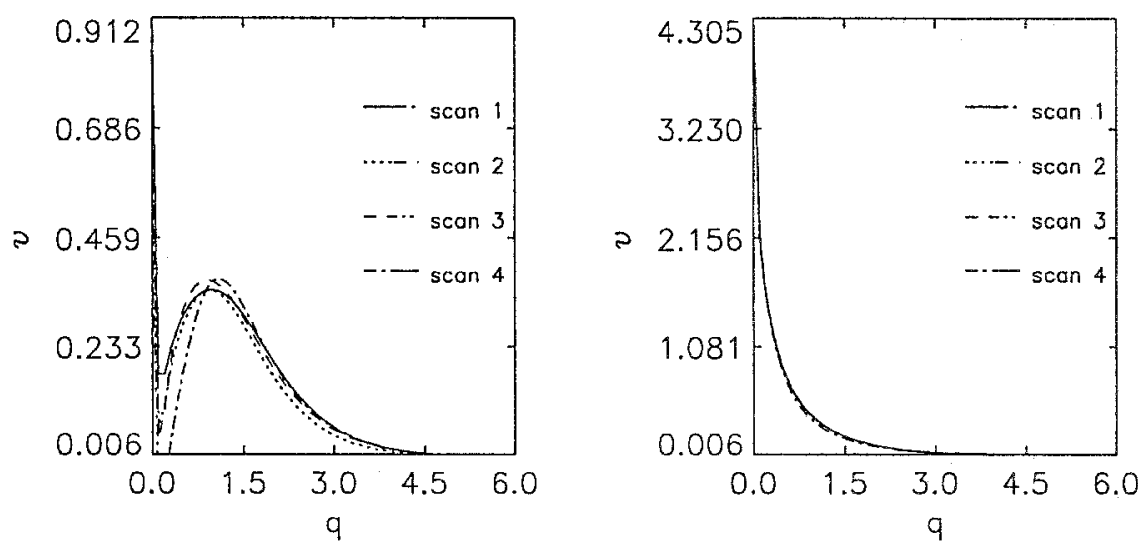

(b)

Fig. 10. Average $\nu(q)$ vs. $q$ for one-dimensional deriv 1 (left) and deriv2 (right) dissipations. (a) $\backslash t$, and (b) $\chi_{x}$. Raw data are used and averaging is performed according to the second to last column in Table I.

TABLE IV

Multifractal spectra parameters for two-dimensional deriv2 dissipation measures and raw data, $\hat{x}_{t}$ and $\hat{\lambda}_{x}$

\begin{tabular}{llllllll}
\hline \multirow{2}{*}{ Scan } & $\hat{\chi}_{\mathrm{t}}$ & & & & $\hat{\chi}_{x}$ \\
\cline { 2 - 3 } \cline { 6 - 8 } & $\nu$ & $\alpha_{1}$ & $D_{2}$ & & & \\
\hline 1 & 3.95 & 1.57 & 1.48 & & 5.55 & 1.27 & 0.99 \\
2 & 3.95 & 1.57 & 1.49 & & 5.37 & 1.27 & 0.98 \\
3 & 3.94 & 1.57 & 1.48 & & 5.33 & 1.27 & 0.98 \\
4 & 3.87 & 1.56 & 1.46 & & 5.12 & 1.33 & 1.07 \\
\hline
\end{tabular}



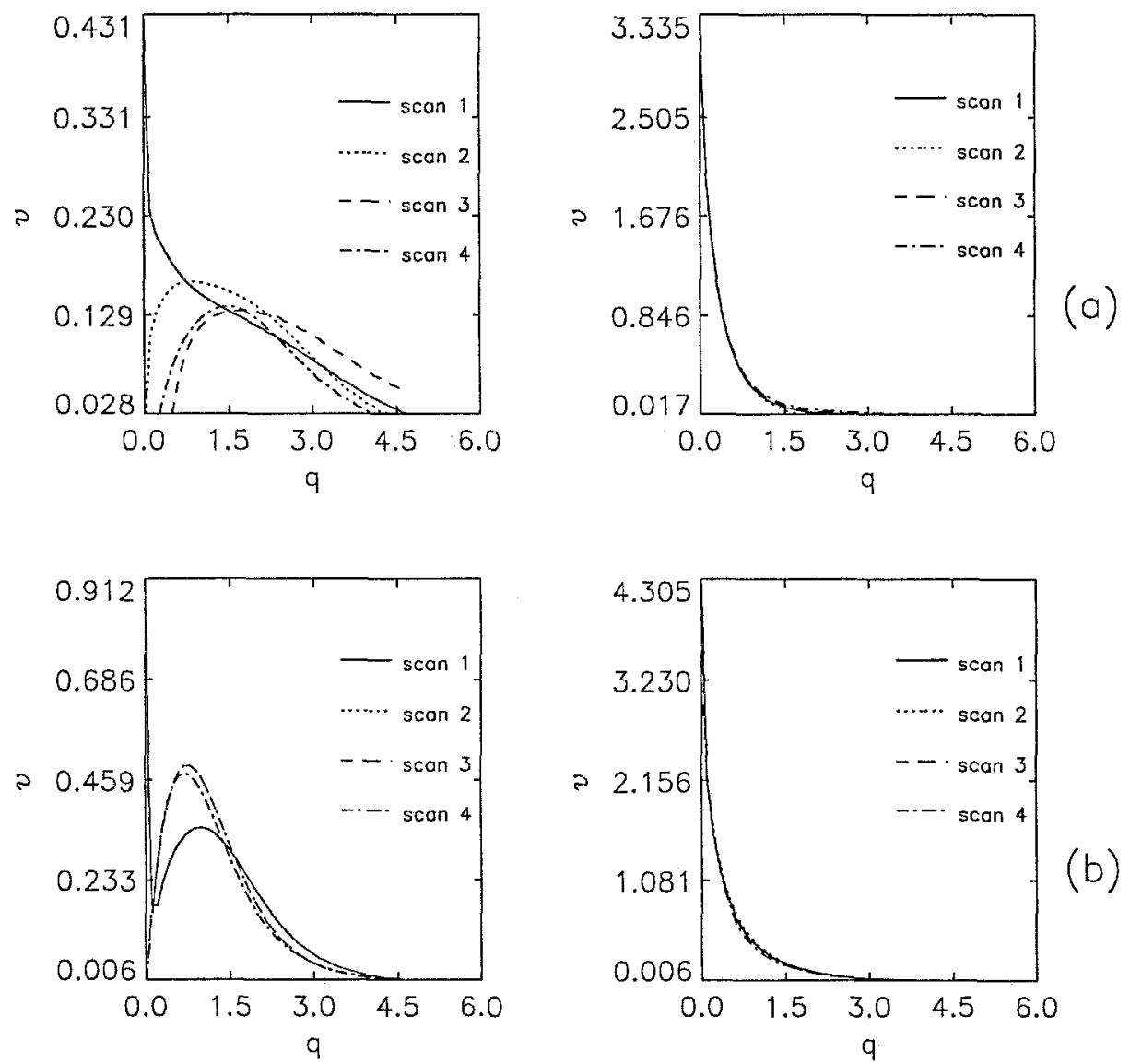

(b)

Fig. 11. Average $\nu(q)$ vs. $q$ for one-dimensional deriv 1 (left) and deriv 2 (right) dissipations. (a) $\lambda_{t}$, and (b) $\chi_{x}$. Data in space are transformed according to wind velocities and averaging is performed according to the last column in Table I.

\section{Conclusions}

An analysis carried out on a statistical measure which approximates the scalar dissipation rate of water vapour content (one-dimensional profiles and two-dimensional time-space measures) shows that a multifractal formalism provides a convenient framework for the description of turbulence in the boundary layer. The most important parameters of the computed multifractal spectra (intermittency exponent $\nu$, entropy dimension $\alpha_{1}$, and correlation dimensions $D_{2}$ ) showed that the water vapour dissipation rate, $\chi$, is more intermittent than the energy dissipation rate, $\epsilon$, in agreement with previously published results (Sreenivasan, 1991). Even though current models such as the $p$ - and $\alpha$-models appear to capture the intrinsic variability in the energy dissipation rate, they can not be used to describe the intermittency exhibited in our scalar gradient rates. The characteristics of the multifractal spectrum, $f(\alpha)$, and the intermittency function, $\nu(q)$, suggest that the 

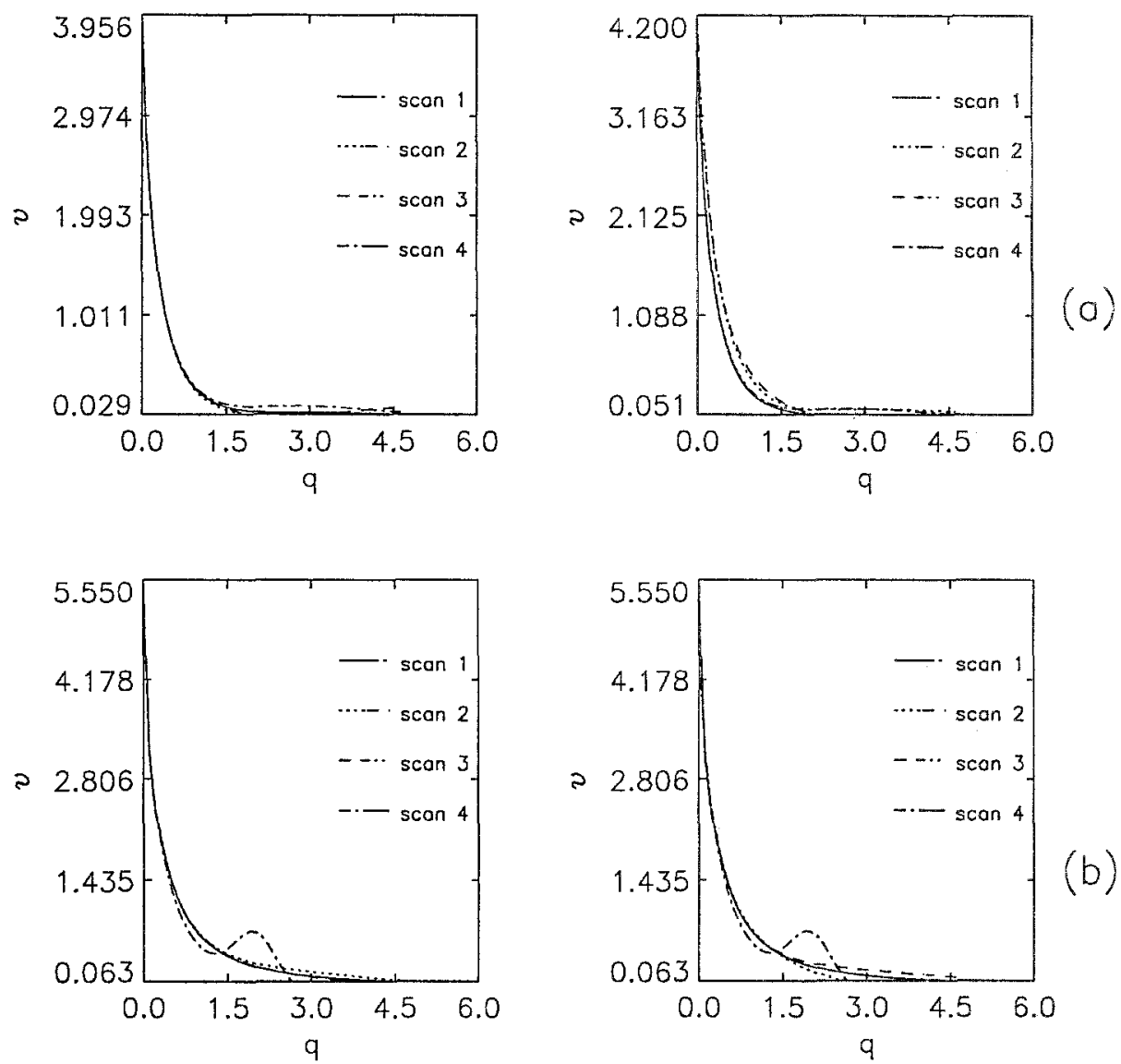

Fig. 12. $\nu\{q\}$ vs. $q$ for two-dimensional deriv2 dissipations. Raw data (left), transformed data (right). (a) $\hat{\chi}_{t}$, and (b) $\hat{\chi}_{x}$.

universal multifractal model of Schertzer and Lovejoy (1987) may be used for modeling the observed specific humidity intermittencies.

The remarkable similarity that exists among all multifractal spectra found from raw or transformed (using relevant velocities) data under all alternative stability conditions implies that for the water vapour dissipation: (a) Taylor's hypothesis could be used in two-dimensional analysis, and (b) prevailing atmospheric stability conditions appear to be irrelevant for the study of interwoven events. Dissipation rates at fixed times $\left(\chi_{x}\right.$ and $\hat{\chi}_{x}$ ) exhibit a little more intrinsic variability (intermittency) than those at fixed locations $\left(\chi_{t}\right.$ and $\left.\hat{\chi}_{t}\right)$ irrespectively of the prevailing atmospheric stability conditions. More experiments are needed to investigate these features.

Markedly different multifractal spectra were observed when using alternative methods of gradient (dissipation) calculations, indicating that a proper definition of the measure being analyzed is needed for accurate description of turbulence. The 
use of a conservative cascade, deriv2, resulted in a consistent geometric definition of dissipation, whose results were nicely reproduced by an existing model. The use of a mathematically correct expression coupled with squaring, deriv1, leads to a nonlinear cascade which gave curvatures on the intermittency function which were not matched by any of the most popular models. We propose deriv 2 as the method to be used to calculate dissipation rates, but suggest that the additional information given by deriv 1 should also be considered. Extensions of the theories to account for such alternative definitions should be made.

\section{Acknowledgements}

We thank Dr. Gabriel G. Katul for his assistance with the experimental setup. Also, we thank Professor Joan Ziser for helpful editorial comments. The valuable comments of anonymous reviewers are appreciated. This work was supported in part by the Kearney Foundation of Soil Science and by the National Science Foundation.

\section{References}

Anselmet, F., Gagne, Y., Hopfinger, E. J., and Antonia, R. A.: 1984, 'High-Order Velocity Structure Functions in Turbulent Shear Flows', J. Fluid Mech. 140, 63-89.

Borgas, M. S.: 1992, 'A Comparison of Intermittency Models in Turbulence', Phys. Fluids A 4(9), 2055-2061.

Chhabra, A. B. and Jensen, R.: 1989, 'Direct Determination of the $f(\alpha)$ Singularity Spectrum', Phys. Rev. Lett. 62, 1327-1330.

Chhabra, A. B. and Sreenivasan, K. R.: 1991a, 'Probabilistic Multifractals and Negative Dimensions', in L. Sirovich (ed.), New Perspectives in Turbulence, Springer-Verlag, New York, pp. 271-288.

Chhabra, A. B. and Sreenivasan, K. R.: 1991b, 'Negative Dimensions: Theory, Computation, and Experiment', Physical Review A 43(2), 1114-1117.

Cooney, J., Petri, K., and Salik, A.: 1985, 'Measurement of High Resolution Atmospheric Watervapour Profiles by Use of a Solar-Blind, Raman-Lidar', Appl. Optics. 24, 104-108.

Eichinger, W. E., Cooper, D. I., Parlange, M. B., and Katul, G. G.: 1993, "The Application of a Scanning Water Raman-Lidar as a Probe of the Atmospheric Boundary Layer', I.E.E.E. Trans. Geoscience and Remote Sensing 31(1), 70-79.

Eichinger, W. E., Cooper, D. I., Archuletta, F. L., Hof, D. E., Holtkamp, D. B., Karl, R. R., Quick, C. R., and Tiee, J. J.: 1994, 'Development of a Scanning, Solar-Blind, Water Raman-Lidar', Applied Optics 33(18), 3923-3932.

Feder, J.: 1988, Fractals, Plenum Press, New York, 283 pp.

Frisch, U. and Parisi, G.: 1985, 'A Multifractal Model of Intermittency', in M. Ghil, R. Benzi and G. Parisi (eds.), Turbulence and Predictability in Geophysical Fluid Dynamics and Climate Dynamics, North-Holland, Amsterdam, pp. 84-88.

Frisch, U., Sulem, P. L., and Nelkin, M.: 1978, 'A Simple Dynamical Model of Intermittent Fully Developed Turbulence', J. Fluid Mech. 87, 719-724.

Gabriel, P., Lovejoy, S., Schertzer, D., and Austin, G.: 1988, 'Multifractal Analysis of Resolution Dependence in Satellite Imagery', J. Geophys. Res. 15, 1373-1376.

Halsey, T. C., Jensen, M. H., Kadanoff, L. P., Procaccia, I., and Shraiman, B. I.: 1986, 'Fractal Measures and Their Singularities: The Characterization of Strange Sets', Physical Rev. A 33, 1141-1151. 
Hunt, J. C. R., Phillips, O. M., and Williams, D. (eds.): 1991, Turbulence and Stochastic Processes. Kolmogorov's Ideas 50 Years On, The Royal Society, London, $240 \mathrm{pp}$.

Kolmogorov, A. N.: 1941 'The Local Structure of Turbulence in Incompressible Viscous Fluid for' Very Large Reynolds Number', Dokl. Akad. Nauk SSSR 30(4), 399-303.

Kolmogorov, A. N.: 'A Refinement of Previous Hypothesis Concerning the Local Structure of Turbulence of a Viscous Incompressible Fluid at High Reynolds Number', J. Fluid Mech. 13, $82-85$.

Lavallée, D., Lovejoy, S., Schertzer, D., and Ladoy, P.: 1991a, "Nonlinear Variability and Landscape Topography: Analysis and Simulation', in L. De Cola and N. Lam (eds.), Fractals in Geography, Prentice-Hall, pp. 171-205.

Lavallée, D., Lovejoy, S., and Schertzer, D.: 1991b, On the Determination of the Codimension Function', in D. Schertzer and S. Lovejoy (eds.), Non-Linear Variability in Geophysics: Scaling and Fractals, Kluwer, pp. 99-110.

Lovejoy, S. and Schertzer, D.: 1990a, 'Our Multifractal Atmosphere: A Unique Laboratory for Non-Linear Dynamics', Physics in Canada 46(4), 62-71.

Lovejoy, S. and Schertzer, D.: 1990b, 'Multifractals, Universality Classes and Satellite and Radar Measurements of Clouds and Rain Fields', J. Geophys. Res. 95, 2021.

Mandelbrot, B.: 1974, 'Intermittent Turbulence in Self-Similar Cascades: Divergence of High Moments and Dimension of the Carrier', J. Fluid Mech. 62, 331-358.

Mandelbrot, B.: 1983, The Fractal Geometry of Nature, Freeman, New York, 468 pp.

Mandelbrot, B.: 1989, 'Multifractal Measures, Especially for the Geophysicist', in C. H. Sholtz and B. B. Mandelbrot (eds.), Fractals in Geophysics, Birkhauser Verlag, Basel, pp. 5-42.

Mandelbrot, B.: 1990, 'Negative Fractal Dimensions and Multifractals', Physica A, 163, 306-315.

Mandelbrot, B.: 1991, 'Random Multifractals: Negative Fractal Dimensions and the Resulting Limitations of the Thermodynamic Formalism', Proc. R. Soc. Lond. A 434, 79-88.

McCauley, J. L.: 1990, 'Introduction to Multifractals in Dynamical Systems Theory and Fully Developed Fluid Turbulence', Physics Reports 189(5), 225-266.

Meneveau, C. and Sreenivasan, K. R.: 1987, 'A Simple Multifractal Cascade Model for Fully Developed Turbulence', Physical Rev. Letters 59, 1424-1427.

Novikov, E. A. and Stewart, R.: 1964, 'Intermittency of Turbulence and Spectrum of Fluctuations in Energy Dissipation', Izv. Akad. Nauk. SSSR. Ser. Geofiz. 3, 408-412.

Parlange, M. B. and Katul, G. G.: 1992, 'An Advection-Aridity Evaporation Model', Water Resour. Res. 28(1): 127-132.

Puente, C. E., Romero, A. H., Obregón, N., and Vargas, M. F.: 1995, 'Deterministic Multifractals with Negative Dimensions?', J. Hydrology, In press.

Schertzer, D. and Lovejoy, S.: 1983, "Elliptical Turbulence in the Atmosphere', in Proc. of the 4th Symposium on Turbulent Shear Flows, Karlshule, West Germany, pp. 11.1-11.8.

Schertzer, D. and Lovejoy, S.: 1987, 'Physical Modeling and Analysis of Rain and Clouds by Anisotropic Scaling of Multiplicative Processes', J. Geophys. Res. D. 92(8), 9693-9714.

Schertzer, D. and Lovejoy, S.: 1989, 'Nonlinear Variability in Geophysics: Multifractal Analysis and Simulation', in L. Pietronero (ed.), Fractals: Physical Origin and Consequences, Plenum, p. 49.

Sreenivasan, K. R., Prasad, R. R., Meneveau, C., and Ramshankar, R.: 1989, 'The Fractal Geometry' of Interfaces and the Multifractal Distribution of Dissipation in Fully Turbulent Flows', in C. H. Sholtz and B. B. Mandelbrot (eds.), Fractals in Geophysics, Birkhauser Verlag, Basel, pp. $43-60$.

Sreenivasan, K. R.: 1991, "Fractals and Multifractals in Fluid Turbulence', Annu. Rev. Fluid Mech. 23, 539-600.

Sreenivasan, K. R. and Kailasnath, P.: 1993, 'An Update on the Intermittency Exponent in Turbulence', Phys. Fluids A 5(2), 512-514.

Sirovich, L. (ed.): 1991, New Perspectives in Turbulence, Springer-Verlag, New York, 367 pp.

Taylor, G. I.: 1938, 'The Spectrum of Turbulence', Proc. Roy. Soc. A 164(919), 476-490.

Tessier, Y., Lovejoy, S., and Schertzer, D.: 1993a, 'Universal Multifractals: Theory and Observations for Rain and Clouds', J. Appl. Meteorol. 32: 223-250.

Tessier, Y., Lovejoy, S., Schertzer, D., Lavallée, D., and Kerman, B.: 1993b, 'Universal Multifractal Indices for the Ocean Surface at Far Red Wavelengths', Geophys. Res. Lett. 20(12), 1167-1170. 
Wilson, J., Schertzer, D., and Lovejoy, S.: 1991, 'Continuous Multiplicative Cascade Models of Rain and Clouds', in D. Schertzer and S. Lovejoy (eds.), Non-Linear Variability in Geophysics: Scaling and Fractals, Kluwer, pp. 185-207. 